\title{
Knowledge and Perceived Barriers towards Intermittent Prevention of Malaria in Pregnancy: A Cross-Sectional Study
}

\author{
Frank Bediako Agyei ${ }^{1}$, Gideon Dzando ${ }^{*}{ }^{(1)}$, Anthony B. Donyi ${ }^{1}$, Elisha A. Nonoh ${ }^{3}$, \\ Rebecca Dordunu", Catherine Konadu Opoku ${ }^{5}$
}

\author{
${ }^{1}$ Department of Nursing, Presbyterian University College, Ghana, Abetifi-Kwahu, Ghana \\ ${ }^{2}$ College of Nursing and Health Sciences, Flinders University, Bedford Park, South Australia, Australia \\ ${ }^{3}$ Holy Family Nursing and Midwifery Training College, Berekum, Ghana \\ ${ }^{4}$ School of Nursing and Midwifery, University of Ghana, Legon, Ghana \\ ${ }^{5}$ University of Ghana Business School, University of Ghana, Legon, Ghana \\ Email: `gdzando@gmail.com
}

How to cite this paper: Agyei, F.B., Dzando, G., Donyi, A.B., Nonoh, E.A., Dordunu, R. and Opoku, C.K. (2021) Knowledge and Perceived Barriers towards Intermittent Prevention of Malaria in Pregnancy: A Cross-Sectional Study. Open Journal of Internal Medicine, 11, 27-38.

https://doi.org/10.4236/ojim.2021.111002

Received: February 11, 2021

Accepted: March 21, 2021

Published: March 24, 2021

Copyright (c) 2021 by author(s) and Scientific Research Publishing Inc. This work is licensed under the Creative Commons Attribution International License (CC BY 4.0).

http://creativecommons.org/licenses/by/4.0/

\begin{abstract}
Background: Intermittent prevention of malaria is a key strategy adopted globally to ensure the wellbeing of pregnant women. By extension, these strategies enhance the healthy development of the fetus. This study assesses the knowledge and practices of pregnant women towards intermittent malaria prevention strategies in the Juaben Government Hospital of Ghana. Methods: A cross-sectional, quantitative, descriptive survey was conducted among 120 pregnant women at the antenatal clinic of the Juaben Government hospital in the Ashanti region of Ghana using a simple random sampling technique in selecting the study participants. The participant response rate was 94.5\%. Data were analyzed using Statistical Package for the Social Sciences (SPSS) version 22.0. Results: Majority (54.2\%) of respondents were between 20 - 29 years of age. Majority of study respondents attended Antenatal visits in their first trimester of pregnancy. Majority (69.2\%) have knowledge of malaria preventive strategies in pregnancy, with about $70 \%$ of respondents stating these strategies were designed only for pregnant women. Only $37.5 \%$ of study respondents could rightly identify the chemoprophylaxis indicated for malaria prevention in pregnancy. Whereas majority (93.3\%) of respondents owned insecticide-treated mosquito nets, only $63.3 \%$ utilized these nets for their purpose. Barriers to utilization of insecticide-treated nets include difficulty in setting up the nets (26.7\%), using mosquito coils (10.8\%), and feeling uncomfortable sleeping in the nets (36.7\%). Barriers to taking malaria prophylaxis include distance to health facilities (28.3\%), thoughts of not being sick with malaria (55.8\%), using herbs (10.0\%), and not being aware of the essence
\end{abstract}


of prophylaxis (7.5\%). Conclusion: Knowledge of malaria preventive strategies in pregnancy is high. However, utilization of these strategies is low due to perceived human and institutional barriers. A comprehensive community-wide approach is required to improve the utilization of these services among pregnant women.

\section{Keywords}

Malaria, Intermittent Prevention, and Treatment (IPTp), Insecticide Treated Nets (ITNs), Antenatal Care, Sulphadoxine Pyrimethamine (SP)

\section{Introduction}

Malaria is an enormous public health problem that affects people of all ages. The World Health Organization (WHO) identifies malaria as a common problem in five regions globally. Sub-Saharan Africa has the largest burden of malarial disease, with over $90 \%$ of the world's malaria-related deaths occurring in this region [1].

Globally, 125 million women are at risk of malaria in pregnancy and approximately $19.7 \%$ of all stillbirths are attributed to malaria in pregnancy [2].

The World Health Organization estimates that about 219 million cases and 435,000 malaria-related deaths occur globally each year [3]. Acute symptomatic malaria accounts for majority of premature deliveries resulting from maternal anaemia. This in effect disrupts the overall developmental processes of the unborn child [4] [5] and threatens the socio-economic fortunes of individuals, families, and their communities at large.

Malaria is a major cause of illness and death in Ghana, particularly among children and pregnant women. In 2012 for instance, malaria accounted for about $38.8 \%$ of all hospital admissions, and $38.9 \%$ of all out-patient illnesses [6]. The Ghana Health Service reported in 2015 that malaria is hyperendemic in Ghana and among pregnant women, it accounted for $17.6 \%$ of the outpatients' department (OPD) attendance, $13.7 \%$ of admissions and $3.4 \%$ of maternal deaths. Malaria in Pregnancy was identified as a major hindrance to the complete attainment of the Millennium Development Goals 4 and 5 in Ghana and had remained a major threat to Sustainable Development Goals 3 and 11.

The World Health Organization recommends a structured and multi-pronged approach to decrease the burden of malaria infection among pregnant women. These are the use of insecticide-treated nets (ITNs), the use of Intermittent Preventive Treatment (IPT), and effective case management of malaria infection [7]. These three processes are not independent of each other and represent key processes that most countries in Sub-Saharan Africa have embedded into their national health policies to ensure the safety of the mother and the unborn child. Intermittent preventive treatment of malaria in pregnancy (IPTp) is the full therapeutic course of antimalarial medicine given to pregnant women at routine 
antenatal care visits, regardless of whether the recipient is infected with malaria. The World Health Organization stipulates that intermittent preventive treatment of malaria in pregnancy reduces maternal malaria episodes, maternal and fetal anaemia, placental parasitaemia, low birth weight, and neonatal mortality [8].

Malaria can be prevented and cured. However, the processes leading to achieving a malaria-free population are costly and limited by the environmental, cultural, and socio-economic conditions of the individuals and communities at risk [9]. Although malaria prevention and treatment services are readily available to pregnant women, the uptake of these services is low in high endemic countries [8]. The preventive and management of malaria in pregnancy require a conscious effort by pregnant women.

This current study seeks to determine the knowledge and perceived barriers towards intermittent prevention of malaria in pregnancy in the Juaben Government hospital.

\section{Materials and Methods}

A descriptive cross-sectional study was carried out among 120 pregnant women who attended antenatal clinics at the Juaben Government Hospital in the Ashanti region of Ghana using a convenient sampling technique. Juaben is a small town in the Ejisu-Juaben municipal district, a district in the Ashanti Region of Ghana. The Juaben hospital is the primary healthcare institution in the municipality. Study participants were randomly selected based on their availability at the time of data collection. Data was collected on the second and fourth Thursdays of June. The second and fourth Thursdays are the main antenatal days in the hospital. Expectant mothers are registered as one of two groups for the main antenatal visits due to their large numbers. Each registrant therefore could only access the main antenatal services ones in every month. The sample size was estimated using Krejcie and Morgan's (1970) [10] sample size determination table. Accordingly, out of the 190 registrants meant to attend the antenatal clinics on both days, 127 respondents were selected. Seven (7) respondents opted out of the study at various stages of the study. From the sitting arrangement at the clinic, study participants were selected from rows numbered in odd numbers.

Survey questionnaires were handed out to study participants who could read and write in English. For those participants who could not read and write, questionnaires were read out in English and translated into the local languages and dialects to facilitate data collection. The translation was done by researchers since they were familiar with the local languages and dialects spoken in the municipality. Survey questions were centred on the use of insecticide-treated mosquito nets and the use of chemoprophylaxis, being the recommended strategies in preventing malaria in pregnancy in Ghana. The malaria control program in Ghana recommends that pregnant women must take at least five (5) doses of SP, commencing at the sixteenth week of gestation. In addition to this, free ITNs are 
distributed to pregnant women at their first antenatal registration as part of the malaria control program. Pregnant women who were not registered or not present at the clinic on the days of data collection were excluded from the study. The instrument for data collection was pretested on 5 pregnant women in Bomfa health centre before the study to ensure validity and reliability.

Data was validated, coded, and analysed using SPSS Version 22.0. Results were presented in descriptive statistics.

\section{Results}

\subsection{Socio-Demographic Characteristics}

A total of 120 pregnant women were interviewed on two separate antenatal days using a structured pretested questionnaire.

Table 1 summarizes the socio-demographic characteristics of study participants. From the table, majority (54.2\%) of respondents were between ages 20 and 29 years. The rests were either less than 20 years $(9.2 \%)$ or more than 29 years (36.6\%). Majority (73.3\%) of study participants were either married or having a partner whiles the rest $(26.7 \%)$ were either divorced or single. Concerning their educational level, majority (46.7\%) had only primary education, $25.8 \%$ had secondary education, 11.7 had tertiary education and $15.8 \%$ had no form of education. With regards to employment, whiles $17.5 \%$ of study participants were unemployed, the majority (82.5\%) were either farmers, traders, artisans, or civil servants. The majority $(60.0 \%)$ of study participants belong to the Akan ethnic group while the rest $40 \%$ were either Fantes, Ewes, Gas, or from other minor ethnic groups. Christians dominated the study, forming a (96.0\%) majority while the rest (40\%) belonged to the Islamic faith. The majority (44.2\%) of the women had two children, $15.8 \%$ had only one child, $29.2 \%$ had three or more children whiles $10.8 \%$ were presenting with their first pregnancies. Majority of the study participants were in their first trimester of pregnancy whiles the rest (47.5\%) were either in their second or third trimesters. Concerning pre-existing medical conditions, only $5.8 \%$ of the women had diabetes and $4.2 \%$ had hypertension. The rest $90 \%$ had no known pre-existing medical condition.

\subsection{Knowledge on Intermittent Prevention and Treatment of Malaria}

From Table 2, more than half $(69.2 \%)$ of respondents have heard about IPTp whiles the rest $30.8 \%$ had no knowledge on IPTp. The majority $(65.8 \%)$ of respondents knew the purpose of IPTp, whiles the rest (34.2\%) held contrary opinions. Whereas majority $(70.0 \%)$ believed IPTp-SP was meant for pregnant women, the rest $30 \%$ were of the view that the schedule was meant for either infants, the aged, or men. On the drug of choice currently used for IPTp, only $37.5 \%$ knew the drug in use. The rest mentioned Chloroquine (26.7\%), Artesunate (20.0\%), and Quinine (15.8\%). Concerning the right time during pregnancy for commencing malaria prophylaxis, $11.7 \%$ of study participants did not know 
when this regimen is commenced, $23.3 \%$ chose the first trimester, $18.3 \%$ chose the third trimester, and the majority (46.7\%) chose the second trimester. Regarding malaria education at Antenatal clinics, a majority (61.7\%) attested to malaria education being held at Antenatal clinics whiles $38.3 \%$ had a contrary opinion. Regarding the side effects of malaria prophylaxis, whiles $11.7 \%$ did not know about any side effects, the rest (88.3\%) noted either nausea, vomiting, dizziness, or bodily weakness as some of the side effects.

\subsection{Barriers to the Use of Intermittent Preventive Strategies}

The majority (93.3\%) of respondents personally owned insecticide-treated mosquito nets while (6.7\%) did not have any. On the usage, majority (63.3\%) attested to using ITNs and $36.7 \%$ did not use ITNs. Regarding the reasons for non-usage of ITNs during pregnancy, $26.7 \%$ of respondents said they had difficulty setting up the nets, $36.7 \%$ said the nets generated too much heat, $10.8 \%$ preferred to use mosquito coils, $22.5 \%$ felt uncomfortable sleeping in the nets and $7.5 \%$ were concerned with the chemicals used to treat the nets. Concerning reasons for not taking SP, $46.7 \%$ of participants preferred to sleep under ITNs, $55.8 \%$ said because they were not sick with malaria, $28.3 \%$ attributed non-usage to distance to health facility, $37.5 \%$ were afraid of possible side effects, $10.0 \%$ preferred to use herbs, $7.5 \%$ were unaware of SP. None of the respondents blamed non-usage to unavailability of SP in health facilities. Regarding what motivates participants to complete their SP doses, $80.8 \%$ wanted to be directly observed, $90.8 \%$ wanted fewer doses, $93.3 \%$ said they needed more education, $65.0 \%$ said not walking long distance to get SP, $80.8 \%$ said they needed a better taste of the medications.

Table 1. Socio-demographic characteristics of study participants $(\mathrm{N}=120)$.

\begin{tabular}{|c|c|c|}
\hline Characteristics & Frequency $(\mathrm{N})$ & Percentage (\%) \\
\hline \multicolumn{3}{|l|}{ Age group } \\
\hline Below 20 & 11 & 9.2 \\
\hline $20-29$ & 65 & 54.2 \\
\hline $30-39$ & 37 & 30.8 \\
\hline $40-49$ & 7 & 5.8 \\
\hline \multicolumn{3}{|l|}{ Marital Status } \\
\hline Single & 28 & 23.3 \\
\hline Married/Co-habiting & 88 & 73.3 \\
\hline Divorced & 4 & 3.3 \\
\hline Widowed & 0 & 0 \\
\hline \multicolumn{3}{|l|}{ Educational level } \\
\hline no formal education & 19 & 15.8 \\
\hline
\end{tabular}




\section{Continued}

\begin{tabular}{ccc}
\hline primary & 56 & 46.7 \\
secondary & 31 & 25.8 \\
tertiary & 14 & 11.7 \\
\hline Occupation & 21 & 17.5 \\
Unemployed & 16 & 13.3 \\
Farming & 42 & 35.0 \\
Trading & 28 & 23.3 \\
Artisanship & 13 & 10.8 \\
Civil servant & & \\
Ethnicity & 72 & 60.0 \\
Akan & 11 & 9.2 \\
Ewe & 8 & 6.7 \\
Ga & 13 & 10.8 \\
Fante & 16 & 13.3 \\
Other & &
\end{tabular}

Religion

\begin{tabular}{ccc} 
Christianity & 96 & 80.0 \\
Islamic & 24 & 20.0 \\
\hline Number of children & 19 & 15.8 \\
One & 53 & 44.2 \\
Two & 24 & 20.0 \\
Three & 11 & 9.2 \\
More than three & 13 & 10.8 \\
None & & \\
1st & 63 & 52.5 \\
2nd & 45 & 37.5 \\
3rimester of first ANC & 12 & 10.0 \\
\hline
\end{tabular}

Do you have any of these disease

$\begin{array}{lll}\text { Diabetes } & 7 & 5.8\end{array}$

$\begin{array}{ccc}\text { Hypertension } & 5 & 4.2 \\ \text { Sickle cell disease } & 0 & 0 \\ \text { G6PD } & 0 & 0 \\ \text { None } & 108 & 90.0\end{array}$


Table 2. Participants' knowledge of IPTp -SP $(\mathrm{N}=120)$.

\begin{tabular}{|c|c|c|}
\hline Variable & $\mathbf{N}$ & $\%$ \\
\hline \multicolumn{3}{|l|}{ Have you heard about IPTp } \\
\hline Yes & 83 & 69.2 \\
\hline No & 37 & 30.8 \\
\hline \multicolumn{3}{|l|}{ What is IPT-Sp? } \\
\hline Taking of SP to prevent malaria in pregnancy & 79 & 65.8 \\
\hline Taking of drugs when you are pregnant & 28 & 23.4 \\
\hline Taking drug supplements & 13 & 10.8 \\
\hline \multicolumn{3}{|l|}{ Who can be given IPTp-SP? } \\
\hline Pregnant women & 84 & 70.0 \\
\hline Infant & 12 & 10.0 \\
\hline Aged people & 15 & 12.5 \\
\hline Men & 9 & 7.5 \\
\hline \multicolumn{3}{|l|}{ Drug of choice for IPTp } \\
\hline Sulfadoxine Pyrimethamine (SP) & 45 & 37.5 \\
\hline Artesunate single dose & 24 & 20.0 \\
\hline Chloroquine & 32 & 26.7 \\
\hline Quinine & 19 & 15.8 \\
\hline \multicolumn{3}{|l|}{ Trimester for going for SP } \\
\hline First trimester (1st - 3rd months) & 28 & 23.3 \\
\hline Second trimester (4th - 6th months) & 56 & 46.7 \\
\hline Third trimester (7th - 9th months) & 22 & 18.3 \\
\hline Don't know & 14 & 11.7 \\
\hline \multicolumn{3}{|c|}{ Is malaria a topic often discussed during ante-natal health talks? } \\
\hline Yes & 74 & 61.7 \\
\hline No & 46 & 38.3 \\
\hline \multicolumn{3}{|l|}{ Side effects of IPT } \\
\hline Nausea & 72 & 60.0 \\
\hline Vomiting & 67 & 55.8 \\
\hline Dizziness & 54 & 45.0 \\
\hline Bodily weakness & 23 & 19.2 \\
\hline Don’t know & 14 & 11.7 \\
\hline
\end{tabular}

Table 3. Barriers to the use of intermittent preventive strategies $(\mathrm{N}=120)$.

\begin{tabular}{ccc}
\hline Variable & N & $\%$ \\
\hline Possession of ITN & & \\
Yes & 112 & 93.3 \\
No & 8 & 6.7 \\
\hline
\end{tabular}


Continued

\begin{tabular}{|c|c|c|}
\hline \multicolumn{3}{|l|}{ Use of ITN } \\
\hline Yes & 76 & 63.3 \\
\hline No & 44 & 36.7 \\
\hline \multicolumn{3}{|l|}{ Reasons for not using mosquito nets during pregnancy } \\
\hline Difficulty in setting up & 32 & 26.7 \\
\hline Excessive heat generated under it & 44 & 36.7 \\
\hline Preference for the use of mosquito coil & 13 & 10.8 \\
\hline Uncomfortable to sleep in & 27 & 22.5 \\
\hline Fear of getting sick from the drugs in the net & 9 & 7.5 \\
\hline \multicolumn{3}{|l|}{ Reasons for not taking SP } \\
\hline I sleep under an ITN & 56 & 46.7 \\
\hline I was not sick with malaria & 67 & 55.8 \\
\hline The health unit is far & 34 & 28.3 \\
\hline There no drugs at the ANC clinic & 0 & 0 \\
\hline I feared side effects & 45 & 37.5 \\
\hline I use herbs & 12 & 10.0 \\
\hline I am not aware of the use of SP & 9 & 7.5 \\
\hline \multicolumn{3}{|l|}{ Motivate you to correctly complete SP doses } \\
\hline When I am directly observed & 97 & 80.8 \\
\hline Fewer dosage & 109 & 90.8 \\
\hline When I am educated on drug and its usage & 112 & 93.3 \\
\hline When I don't have to walk distance to take it & 78 & 65.0 \\
\hline A better taste of the drug & 97 & 80.8 \\
\hline
\end{tabular}

\section{Discussion}

From the socio-demographic characteristics (Table 1), majority (52.5\%) of the study participants reported for their first antenatal in their first trimester of pregnancy. This collaborates with findings from a similar study conducted in Tanzania by [11] where more than half of the study participants reported for antenatal clinic in their first trimester. Reporting late for Antenatal registration has been identified as a major cause of poor adherence to IPTp-SP regimen in pregnancy [12]. The ministry of health, Ghana, and Ghana health service launched a campaign as part of efforts to make primary healthcare accessible to all Ghanaians. As part of this campaign, The Community-based Health Planning and Services (CHPS) was introduced some fifteen years ago which reduced the burden of traveling long distances to access healthcare. This possibly could account for more than half of study participants reporting early for antenatal services. 
Regarding the level of knowledge of respondents, more than half $(69.2 \%)$ attested to having heard about IPTp-SP. This finding collaborates with findings from studies conducted in Sunyani-Ghana, Nigeria, and Tanzania [13] [14] [15] where majority of study participants knew about IPTp-SP. The high level of knowledge is also evident in Table 2 where more than half of the participants (65.8\%) understood that IPTp-SP included taking medication to prevent malaria, and $70 \%$ understood that the regimen was designed for pregnant women. However, only about $37.5 \%$ were able to identify the drug of choice for malaria prevention in pregnancy. This finding is consistent with findings from a study conducted by [16], where only $31.7 \%$ of respondent were able to identify the complete treatment plan involved in IPTp-SP. This phenomenon is again characterised in this present study where less than half (46.7\%) of the respondents knew the appropriate time to commence malaria prophylaxis in pregnancy. Knowledge of antimalarial medications in Ghana is high possibly because of their availability over the counter and the ability of individuals to identify malaria symptoms without necessarily undergoing any diagnostic investigations as part of required standard practice.

From Table 3, majority (93.3\%) of respondents had treated bedside mosquito nets at home. However, only $63.3 \%$ of respondents used these treated nets. This correlates with findings from a study conducted by [17] in Uganda where although about $84 \%$ of study participants possessed ITNs, only $66 \%$ of these participants use them regularly. With regards to possession, however, findings from both studies are encouraging as these findings exceed the expectation of the World Health organization which pegs possession at about 80\% [18]. Some of the reasons identified by respondents as a cause of non-usage include difficulty in setting up, the heat generated under it, prefers to use mosquito coil, uncomfortable to sleep in, and fear of getting sick from the drugs in the net. These reasons were also found in similar studies in Ethiopia [19] and a trial in Kenya which assessed the reasons for non-usage of ITNs the previous night Kenya as part of an intervention program to enhance coverage [20]. In Ghana, insecticide-treated mosquito nets are often given to households at no cost as directed by the World Health Organization as part of efforts to eradicate malaria. This might explain why such many study participants have these nets at home.

Regarding the use of SP in pregnancy, this current study found distance to a health facility, not being sick with malaria, using herbs, already using ITN, and not being aware of SP as malaria prophylaxis. This finding is consistent with findings from a study conducted by [21] in northern Nigeria where non-adherent to SP regimen was identified among pregnant women. In both studies, however, majority of study participants preferred to take their chemoprophylaxis when they were directly observed. In its inception, the World Health Organization recommended that direct observation therapy should be implored to enhance wider coverage of SP usage by pregnant women [22]. For the women who were willing to take SP if doses were fewer, their assertion could boost a wide coverage and uptake of SP since most women in sub-Saharan Africa do not go for Ante- 
natal visits until after the second trimester [23]. To achieve a wider coverage of preventing malaria in pregnancy, policymakers must target and streamline programmes that involve families and communities at large instead of limiting resources to pregnant women alone. As seen in Table 2 and Table 3, majority of the women, although knew about malaria prevention, they still required a certain level of motivation to start or adhere to these preventive strategies. Contrary to findings from [24], which identified structural factors such as low medication stock and distance to health facilities as major challenges to the implementation of malaria prevention programs, findings from this present study found otherwise, with none of the respondents attributing poor compliance to non-availability of medications and supplies in health facilities.

\section{Conclusion}

Malaria in Pregnancy is a public health canker that adversely affects pregnant women, children, and their families in Sub-Saharan Africa. Knowledge of preventive strategies coupled with the availability of logistics is an important step in achieving a wider coverage of malaria prevention programs. Early antenatal visits and the motivation to frequently attend these visits are important to ensure that pregnant women receive complete doses of their chemoprophylaxis. Health education at antenatal clinics is also important to ensure that pregnant women are using their mosquito nets as required of them.

\section{Limitations}

The study was conducted on specific antenatal days of the month and pregnant women who were not present were excluded from the study. Also, this survey utilized closed-ended questionnaires which did not make provision to explore the concept further as study participants limited their responses to specific questions.

\section{Future Directions}

The researchers recommend that further studies should be conducted in multiple sites, including the communities to involve a larger group as well as to understand the variations of responses that may exist among diverse populations to inform policy and practice.

\section{Ethics}

Ethical approval for the study was obtained from the Department of Nursing of the Presbyterian University College, Ghana, and the Management Board of the Juaben Government Hospital. Participation in the study was by choice, and codes were used to identify respondents instead of names. The process and purpose of the study were explained to respondents at the start of data collection. Participants were encouraged to opt-out at any time during the process when they wished to. 


\section{Acknowledgements}

We wish to acknowledge the efforts of our study participants for taking their time in answering the questionnaires. We also acknowledge the contribution of the staff and management of Juaben Government Hospital for their warm reception throughout this study.

\section{Conflicts of Interest}

The authors declare no conflict of interest in preparing and publishing this paper.

\section{References}

[1] WHO (2016) World Malaria Report. World Health Organization, Geneva.

[2] Lawn, J.E., Blencowe, H., Waiswa, P., Amouzou, A., Mathers, C., Hogan, D., Draper, E.S., et al. (2016) Stillbirths: Rates, Risk Factors, and Acceleration towards 2030. The Lancet, 387, 587-603. https://doi.org/10.1016/S0140-6736(15)00837-5

[3] WHO (2018) World Malaria Report 2018. World Health Organization, Geneva. https://www.who.int/malaria/publications/world-malaria-report-2018/en

[4] Park, S., Nixon, C.E., Miller, O., Choi, N.K., Kurtis, J.D., Friedman, J.F. and Michelow, I.C. (2020) Impact of Malaria in Pregnancy on Risk of Malaria in Young Children: Systematic Review and Meta-Analyses. The Journal of Infectious Diseases, 222, 538-550. https://doi.org/10.1093/infdis/jiaa139

[5] Fried, M. and Duffy, P.E. (2017) Malaria during Pregnancy. Cold Spring Harbor Perspectives in Medicine, 7, a025551. https://doi.org/10.1101/cshperspect.a025551

[6] Awine, T., Malm, K., Bart-Plange, C. and Silal, S.P. (2017) Towards Malaria Control and Elimination in Ghana: Challenges and Decision-Making Tools to Guide Planning. Global Health Action, 10, Article ID: 1381471. https://doi.org/10.1080/16549716.2017.1381471

[7] WHO (2004) A Strategic Framework for Malaria Prevention and Control during Pregnancy in the Africa Region. World Health Organization Regional Office for Africa, Geneva, AFR/MAL/04/01.

[8] Global Malaria Programme: Pregnant Women and Infants. World Health Organization. http://apps.who.int/malaria/pregnantwomenand infants.html

[9] Fokam, E.B., Ngimuh, L., Anchang-Kimbi, J.K. and Wanji, S. (2016) Assessment of the Usage and Effectiveness of Intermittent Preventive Treatment and Insecticide-Treated Nets on the Indicators of Malaria among Pregnant Women Attending Antenatal Care in the Buea Health District, Cameroon. Malaria Journal, 15, 172. https://doi.org/10.1186/s12936-016-1228-3

[10] Krejcie, R.V. and Morgan, D.W. (1970) Determining Sample Size for Research Activities. Educational and Psychological Measurement, 30, 607-610.

https://doi.org/10.1177/001316447003000308

[11] Anders, K., Marchant, T., Chambo, P., Mapunda, P. and Reyburn, H. (2008) Timing of Intermittent Preventive Treatment for Malaria during Pregnancy and Implications of Current Policy on Early Up Take in North-East Tanzania. Malaria Journal, 7, 79. https://doi.org/10.1186/1475-2875-7-79

[12] Oyibo, W.A. and Agomo, C.O. (2011) Scaling Up of Intermittent Preventive Treatment of Malaria in Pregnancy Using Sulphadoxine-Pyrimethamine: Prospects and 
Challenges. Maternal and Child Health Journal, 15, 542-552. https://doi.org/10.1007/s10995-010-0608-5

[13] Ibrahim, H., Maya, E.T., Issah, K., Apanga, P.A., Bachan, E.G. and Noora, C.L. (2017) Factors Influencing Uptake of Intermittent Preventive Treatment of Malaria in Pregnancy Using Sulphadoxine Pyrimethamine in Sunyani Municipality, Ghana. The Pan African Medical Journal, 28, 122. https://doi.org/10.11604/pamj.2017.28.122.12611

[14] Amoran, O.E., Ariba, A.A. and Iyaniwura, C.A. (2012) Determinants of Intermittent Preventive Treatment of Malaria during Pregnancy (IPTp) Utilization in a Rural Town in Western Nigeria. Reproductive Health, 9, Article No. 12.

https://doi.org/10.1186/1742-4755-9-12

[15] Exavery, A., Mbaruku, G., Mbuyita, S., Makemba, A., Kinyonge, I.P. and Kweka, H. (2014) Factors Affecting Uptake of Optimal Doses of Sulphadoxine-Pyrimethamine for Intermittent Preventive Treatment of Malaria in Pregnancy in Six Districts of Tanzania. Malaria Journal, 13, 22. https://doi.org/10.1186/1475-2875-13-22

[16] Obieche, A.O., Enato, E.F. and Ande, A.B. (2015) Assessment of Knowledge, Interventional Practices for, and Impact of Malaria in Pregnancy among Parturient Women in a Nigerian Tertiary Healthcare Facility. Tropical Journal of Pharmaceutical Research, 14, 1103-1110. https://doi.org/10.4314/tjpr.v14i6.24

[17] Taremwa, I.M., Ashaba, S., Adrama, H.O., Ayebazibwe, C., Omoding, D., Kemeza, I., Hilliard, R., et al. (2017) Knowledge, Attitude and Behaviour towards the Use of Insecticide Treated Mosquito Nets among Pregnant Women and Children in Rural Southwestern Uganda. BMC Public Health, 17, 794. https://doi.org/10.1186/s12889-017-4824-4

[18] WHO (2007) Insecticide-Treated Mosquito Nets: WHO Position Statement. http://www.ivcc.com/sites/ivcc.mrmdev.co.uk/files/content/itnspospaperfinal.pdf

[19] Baume, C.A., Reithinger, R. and Woldehanna, S. (2009) Factors Associated with Use and Non-Use of Mosquito Nets Owned in Oromia and Amhara Regional States, Ethiopia. Malaria Journal, 8, 264. https://doi.org/10.1186/1475-2875-8-264

[20] Alaii, J.A., Hawley, W.A., Kolczak, M.S., Ter Kuile, F.O., Gimnig, J.E., Vulule, J.M., Phillips-Howard, P.A., et al. (2003) Factors Affecting Use of Permethrin-Treated Bed Nets during a Randomized Controlled Trial in Western Kenya. The American Journal of Tropical Medicine and Hygiene, 68, 137-141.

https://doi.org/10.4269/ajtmh.2003.68.137

[21] Iliyasu, Z., Gajida, A.U., Galadanci, H.S., Abubakar, I.S., Baba, A.S., Jibo, A.M. and Aliyu, M.H. (2012) Adherence to Intermittent Preventive Treatment for Malaria in Pregnancy in Urban Kano, Northern Nigeria. Pathogens and Global Health, 106, 323-329. https://doi.org/10.1179/2047773212Y.0000000037

[22] Global Malaria Programme, W.H.O. (2012) World Malaria Report 2012.

[23] Hill, J., Hoyt, J., van Eijk, A.M., D’Mello-Guyett, L., Ter Kuile, F.O., Steketee, R., Webster, J., et al. (2013) Factors Affecting the Delivery, Access, and Use of Interventions to Prevent Malaria in Pregnancy in Sub-Saharan Africa: A Systematic Review and Meta-Analysis. PLOS Medicine, 10, e1001488.

https://doi.org/10.1371/journal.pmed.1001488

[24] Diala, C.C., Pennas, T., Marin, C. and Belay, K.A. (2013) Perceptions of Intermittent Preventive Treatment of Malaria in Pregnancy (IPTp) and Barriers to Adherence in Nasarawa and Cross River States in Nigeria. Malaria Journal, 12, 342.

https://doi.org/10.1186/1475-2875-12-342 